\title{
Improved conditions for the aerobic reductive decolourisation of azo dyes by Candida zeylanoides
}

\author{
Patricia A. Ramalho(1) , H. Scholze ${ }^{(2)}$, M. Helena Cardoso ${ }^{(1)}$, M. Teresa Ramalho(2) \\ and A.M. Oliveira-Campos ${ }^{(2)}$
}

Department of Biology ${ }^{(1)}$ and Department of Chemistry ${ }^{(2)}$, University of Minho, Braga, Portugal.

Corresponding author: tramalho@quimica.uminho.pt

Key words: Azo dyes, Methyl orange, Orange II, Yeasts, Decolourisation

\begin{abstract}
A number of anaerobic and aerobic bacterial species are known to decolourise azo dyes through the reduction of the azo bonds, forming the corresponding amines. In this work, we describe improved decolourisation conditions for model azo dyes by the ascomycete yeast Candida zeylanoides. The dyes were derived from the diazonium salts of metanilic and sulfanilic acids and N,N-dimethylaniline or 2-naphthol as coupling components. Total decolourisation times observed in culture media supplemented with $0.2 \mathrm{mM}$ dye ranged from 40 go 60 hours. The initial decolourisation rates were $14-52 \mu$ mol. $(\mathrm{g} \text { dry cell })^{-1} \cdot \mathrm{h}^{-1}$, depending on dye structure. In the course of decolourisation either metanilic acid or sulfanilic acid were detected in the supernatant fluid, showing that decolourization by this yeast strain is due to azo bond reduction. None of those aminobenzenesulphonates supported microorganism growth as carbon and energy source but both could be used, to a limited extent, as nitrogen sources. The azo reductase activity is not significantly affected by pre-adaptation of the microorganism to the dyes.
\end{abstract}

\section{Introduction}

Among the synthetic dyes, which are widely used for textile dyeing and other industrial applications, those containing an azo chromophore constitute the largest class [1]. Since dyes are designed to be resistant to microbial and physico-chemical attack, most of them are not easily destroyed by conventional processes of wastewater treatment, including biological treatment by activated sludge [2,3]. The percentage of unchanged dye after such treatments is estimated to range between 50 and $90 \%$ [4,5], a 
fact which raises environmental problems, not only for obvious aesthetic reasons but also because the dyes themselves, or their biotransformation products, can have deleterious effects on living organisms. Both the structural diversity of dyes and the wide variability in composition of dye-containing effluents are probably decisive factors accounting for the observed recalcitrance of colouring substances [6].

As reviewed by Chung et al. [7], earlier studies on the decolourisation of azo dyes mainly involved anaerobic bacterial species isolated from the intestinal microflora, which cleave the molecules by reduction of the azo bond(s). A closely related research field deals with the investigations on the toxic, mutagenic, or carcinogenic properties of several aromatic amines generated by biological reduction [8,9]. The azo reductase activity detected in bacteria displaying azo bond reduction capabilities has also deserved considerable attention. Azo reductases were isolated from several bacterial sources and some of their molecular properties and substrate specificities were determined $[10,11]$. Some authors have also succeeded in isolating genomic DNA fragments from azoreducing bacterial species and expressing them in non-decolourising species [12-14]. The available evidence indicates that azo reductase activity is associated with different types of genes [12]. In other words, azo reductase activity can be associated with more than one reductase, depending on the particular microorganism and, eventually, on the cultivation conditions.

The most generally accepted mechanism of azo reduction involves the participation of redox mediators, acting as electron shuttles between the extracellular dye and the intracellular reductase. The azo bond reduction step thus occurs extracellularly. Anthrahydroquinone sulfonates [15,16], riboflavin and benzylviologen [17] are examples of redox mediators which have been shown to accelerate azo bond reduction by intact cells. This mechanism also accounts for the reduction of highly polar azo dyes, which can not usually cross cell membranes.

Bacterial azo bond reduction also occurs under aerobic conditions, as demonstrated, about two decades ago $[18,19]$. Since then various reports on anaerobic, aerobic or anaerobic-aerobic sequential treatments for degradation of coloured effluents have appeared, as recently reviewed by Robinson et al. [20] and Stolz [21].

In an earlier report [22], we have provided preliminary evidence on the aerobic azo dye decolourisation by an ascomycete yeast, identified as Candida zeylanoides. In 
the present work we have used modified cultivation conditions in order to improve the decolourisation process by the same microorganism, and investigated some features of the azo reductase activity which mediates dye decolourisation. The utilization of the stable reduction products of the tested dyes by the microorganism was also studied.

\section{Materials and methods}

\section{$2.1 \quad$ Chemicals and culture media components}

The structures of the azo dyes used in this work are represented in table 1. Dyes I-III were synthesized and characterized by conventional methods. Dye IV was purchased from Sigma-Aldrich and used without further purification. Inorganic media components, D-glucose, 3,5-dinitrosalicylic acid (DNS) and methanol, of analytical grade, were obtained from Merck. Tetrabutylammonium phosphate (TBAP), sulfanilic acid and metanilic acid were from Sigma-Aldrich. Complex media components (yeast extract, yeast carbon base (YCB), yeast nitrogen base (YNB) and peptone) were obtained from Difco.

\subsection{Microorganism and maintenance conditions}

The ascomycete yeast C. zeylanoides (strain UM2), was isolated as described in a previous publication [22]. The strain was routinely maintained on slants of a medium containing (\% w/v): glucose (2), peptone (1), yeast extract (0.5), and agar (2).

\subsection{Analytical methods}

Biomass was measured by turbidimetry readings of appropriately diluted culture samples at $640 \mathrm{~nm}$, against a blank prepared with the same dilution of the supernatant in distilled water. The correlation between $O D(640 \mathrm{~nm})$ and cell dry weight $\left(w, \mathrm{~g} . \mathrm{L}^{-1}\right)$ was $O D=1.40 w-0.14\left(r^{2}=0.996\right)$, as experimentally determined by the standard gravimetric method. Dye concentration was estimated by absorbance measurements of supernatant samples, diluted as required, in a buffer of $\mathrm{pH} 4.0$, at dye $\lambda_{\max }$, read against a blank containing the same dilution of buffer in water. Glucose concentration was determined with the DNS reagent [23]. HPLC analyses for detection of dye metabolites were performed in a liquid chromatograph consisting of a pump (model PU-980) and a UV/VIS detector (model UV-975), both from Jasco, and a Chromatopac C-R6A 
recorder, from Shimadzu. The column (Lichrocart 250-4) was packed with Lichrospher100 RP-18, 5 $\mu \mathrm{m}$, from Merck. Sample injection was performed with a $20 \mu$ Rheodyne injector. The eluent composition was 30:70 (v/v) methanol-water, containing $5 \mathrm{mM}$ TBAP. The flow rate was adjusted to $0.6 \mathrm{~mL} \cdot \mathrm{min}^{-1}$ and the separated compounds were detected at $220 \mathrm{~nm}$. Under these conditions the markers for azo bond reduction of dyes I/III and II/IV, metanilic acid and sulfanilic acid, respectively, had retention times of $12.8 \pm 0.3$ and $10.3 \pm 0.3 \mathrm{~min}$. The presence of these compounds in the supernatant fluids was confirmed by using authentic samples as internal standards. Glucose, ethanol and acetic acid concentrations were ocasionally determined in the growth media by HPLC, using a Merck Polyspher OA KC column, with refractive index detection, and arabinose as internal standard [24].

\subsection{Growth and decolourisation assays}

Typical decolourisation experiments were routinely performed in $250 \mathrm{~mL}$ conical flasks with $100 \mathrm{~mL}$ volumes of a sterile medium (normal decolorization medium, NDM) containing (\% w/v): $\left(\mathrm{NH}_{4}\right)_{2} \mathrm{SO}_{4} \quad(0.25)$, yeast extract $(0.25), \quad \mathrm{KH}_{2} \mathrm{PO}_{4} \quad(0.5)$, $\mathrm{MgSO}_{4} .7 \mathrm{H}_{2} \mathrm{O}(0.05), \mathrm{CaCl}_{2} 2 \mathrm{H}_{2} \mathrm{O}(0.013)$ and glucose (2). Concentrated azo dye solutions $(4 \mathrm{mM})$ were separately prepared and filter sterilized (Filtropur $0.2 \mu \mathrm{m})$ and aseptically added to the medium to a final concentration of $0.2 \mathrm{mM}$. The flasks were inoculated with a cell suspension obtained from a freshly grown slant, and incubated at $25^{\circ} \mathrm{C}$, under orbital shaking $(120 \mathrm{rpm})$. Triplicate experiments were run throughout.

In order to elucidate if pre-adaptation of the microorganism to the dyes had any effect on decolorization rates, cultures were started at a high initial cell density (c.a. 1. 3 OD units; $1.0 \mathrm{~g}$ dry cell. $\left.\mathrm{L}^{-1}\right)$. The inocula were prepared from cells in the exponential growth phase, obtained after c.a. $14 \mathrm{~h}$ incubation, in the presence or in the absence of dye, in $100 \mathrm{ml}$ volumes of NDM. Adapted and non-adapted cells were harvested by centrifugation and washed twice before ressuspension in $100 \mathrm{~mL}$ volumes of fresh NDM, containing $0.2 \mathrm{mM}$ dye.

\subsection{Assimilation experiments}

Minimal media (YNB and YCB) were prepared according to the manufactor's instructions and supplemented with $5 \mathrm{mM}$ metanilic or sulfanilic acid for testing these 
compounds as carbon and energy sources or nitrogen sources for the microorganism. Control experiments were performed with $2.5 \mathrm{mM}$ ammonium sulphate as sole nitrogen source, in YCB, or with 5mM glucose as sole carbon and energy source, in YNB.

\section{Table 1.}

Structures and $\lambda_{\max }$ of dyes

Dye
III: $\mathrm{R}=m-\mathrm{RO}=p$-SO3Na $\mathrm{Na}^{\mathrm{d}}$

\section{Results and discussion}

\subsection{Dye effects on the microorganism specific growth rates}

In order to detect possible dye inhibitory effects, separate growth experiments in NDM, containing each one of the dyes I-IV, and a control without dye, were run. As shown in Fig. 1, very similar growth curves were obtained. The observed duplication times ranged between 4.8 and 5.6h, and the biomass, in the stationary phase, reached similar values (5-6 OD units) at $40 \mathrm{~h}$ incubation. These results indicate that, at the concentrations used none of the tested dyes had a significant inhibitory effect on the microorganism growth. 


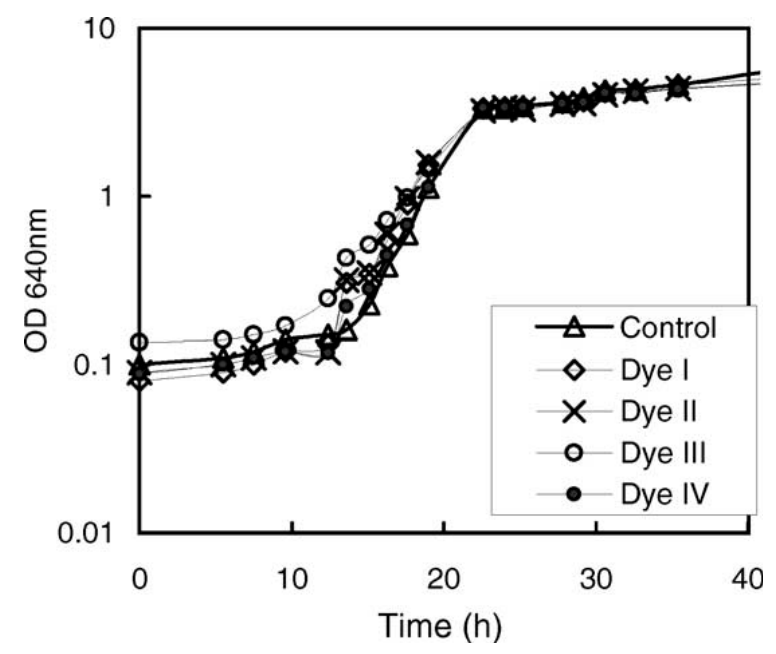

Fig. 1. Growth curves of C. zeylanoides in NDM containing $0.2 \mathrm{mM}$ Dyes I-IV and control growth curve in the same medium without dye.

\subsection{Decolourisation experiments}

As described in a previous report [22], between 10 and $50 \%$ of dyes I-IV (formerly dyes $2 \mathrm{~b}, 2 \mathrm{a}, 1 \mathrm{~b}$ and $1 \mathrm{a}$ ) persisted in the supernatants of shaking cultures of $C$. zeylanoides, after 7 days (168h) of incubation. By using the new culture medium (NDM) it could consistently be observed that, after 40h incubation, the concentration of dyes I and II was undetectable, and that of dyes III and IV was reduced to c.a. $15 \%$ (Fig. 2). The incubation period required for complete decolourisation of the latter dyes was $60 \mathrm{~h}$ (results not shown). In any case, when decolourisation was complete, the yeast cells were also colourless, indicating the absence of any adsorption phenomenon. These results reflect a considerable decrease in the period required for complete decolourisation of these dyes by $C$. zeylanoydes, under conditions that, except for the medium composition, were otherwise similar.

From Fig. 2, it is also apparent that decolourisation is faster during the exponential growth phase of the microorganism, slowing down in the stationary phase. This is not unexpected, since the process must be dependent on cell biomass and on actively growing cells.

The monitoring of $\mathrm{pH}$ revealed a progressive acidification of the culture medium, starting at the onset of the exponential growth phase, which is due to glucose metabolism. Under the conditions described, the presence of ethanol and acetic acid was detected in samples of supernatant fluid collected after c.a. $20 \mathrm{~h}$ and $25 \mathrm{~h}$ incubation, 
respectively (results not shown). The observed $\mathrm{pH}$ variation can, eventually, affect decolourisation rate, as is usually the case for bacterial decolourisation processes $[17,25]$, and also for the chemical azo dye reduction by NADH [26]. However, further experimental evidence will be required in order to draw any conclusion regarding the optimum $\mathrm{pH}$ range for the decolorization process by this yeast strain.
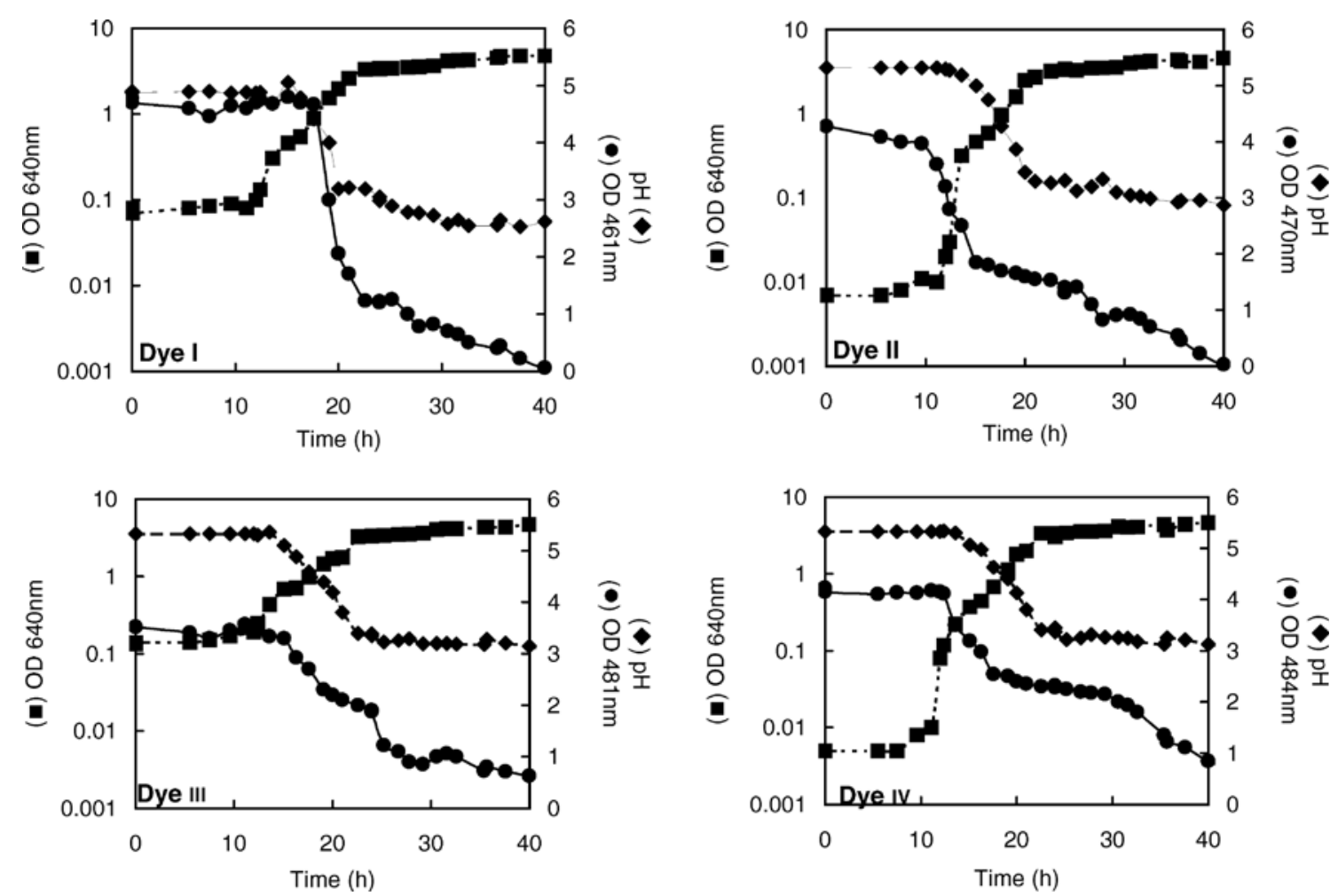

Fig. 2. Time-course of the decolourisation of Dyes I-IV (•),evolution of $\mathrm{pH}(\bullet)$ and cell growth $(\mathbf{\square})$ in batch aerated cultures of C. zeylanoides.

\subsection{Detection of dye reduction products}

HPLC analyses of supernatant samples of dye decolourisation cultures revealed the presence of metanilic acid (for Dyes I and III), or sulfanilic acid (for Dyes II and IV), as illustrated in Fig. 3. These aminobenzenesulfonates are the stable reduction products of the tested dyes and were therefore used as markers for azo bond reduction. The other expected reduction products, 1-amino-2-naphthol and N,N-dimethyl-pphenylenediamine, are quite unstable in aerated solutions. This behaviour has already been described by Kudlich et al. [27] for 1-amino-2-naphthol. The results described above demonstrate that the yeast strain used in the present work cleaves azo bonds by 
reduction, forming the corresponding amines, as observed with decolourising anaerobic or facultative aerobic bacteria.

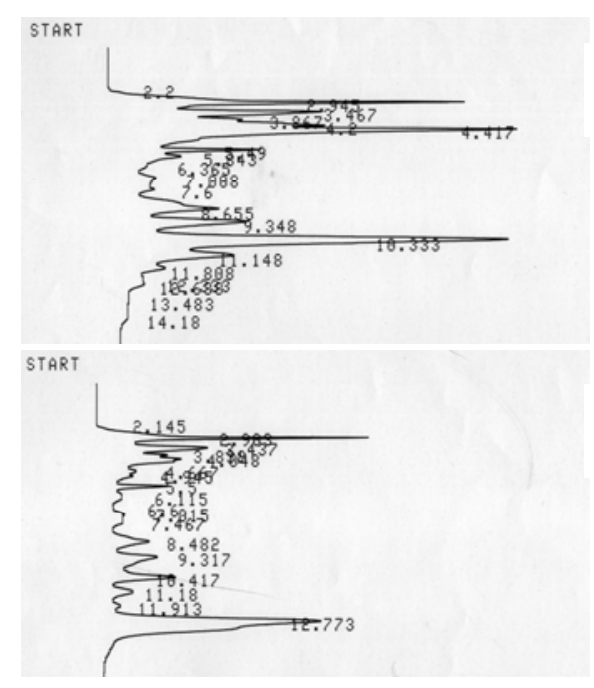

(a)

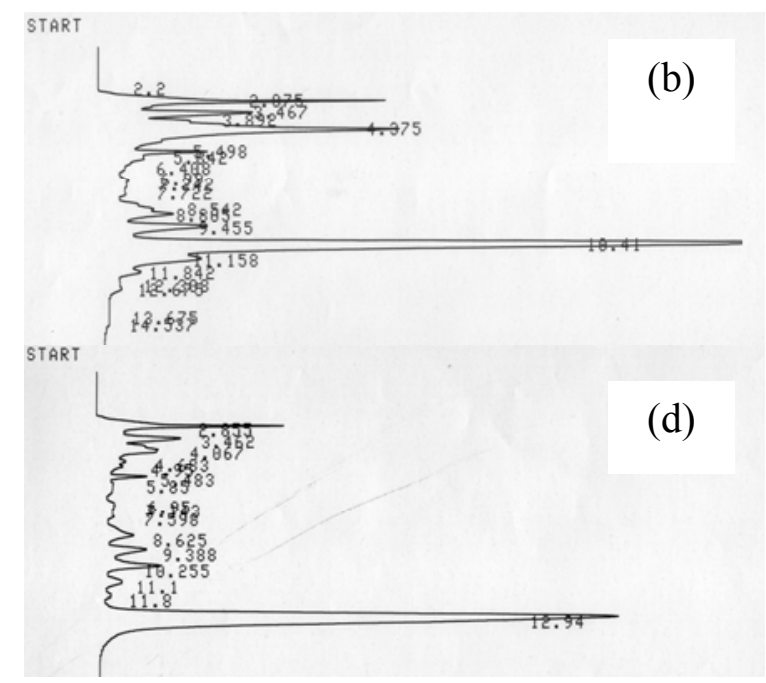

Fig. 3. Chromatograms of supernatant samples taken from growing cultures in media containing Dyes II and III (a and c); chromatograms of the same samples with added sulfanilic and metanilic acids, respectively ( $b$ and $d)$.

\subsection{Assimilation experiments}

Metanilic and sulfanilic acids were separately tested as carbon and energy sources or nitrogen sources by determining specific growth rates of the yeast cells in minimal media (YNB or YCB) supplemented with those compounds, at $5 \mathrm{mM}$ concentrations. Appropriate controls, as described in Section 2, were simultaneously run. The results displayed in Fig. 4 show that the two aminobenzesulphonic acids behave similarly as nitrogen sources, with duplication times of $17 \mathrm{~h}$, much higher than the observed with equimolar inorganic ammonium $(6.7 \mathrm{~h})$. The limited growth observed in YCB can be due to a restricted uptake of the nitrogen sources by the yeast cells or, alternatively, to the formation of some inhibitory product. This aspect is currently being investigated.

In contrast, none of those substrates supported microorganism growth, as carbon and energy source. The sulphonic group occurs rarely in nature and, therefore, degradation mechanisms for sulphonates are not frequent in the microbial world [28]. There are, however, some examples described in the literature relating the 
mineralization of naphthalenesulphonates by a bacterial consortium [29] and of sulfanilic acid by a single bacterial strain, obtained from a mixed culture after extended preadaptation [30]. A similar capability has not been described, so far, in yeast species.

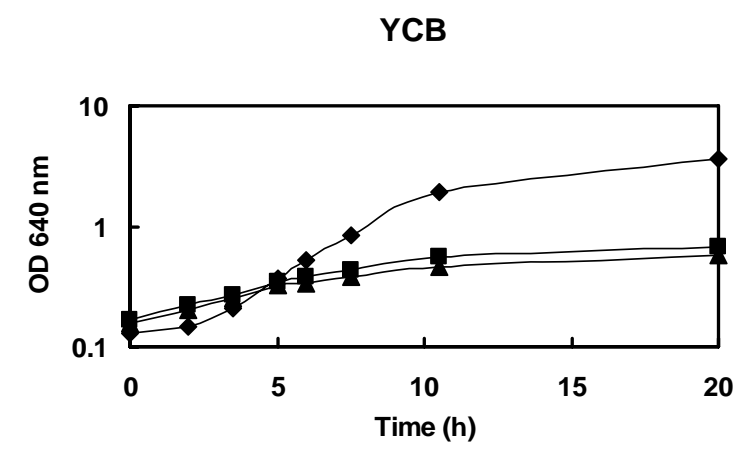

YNB

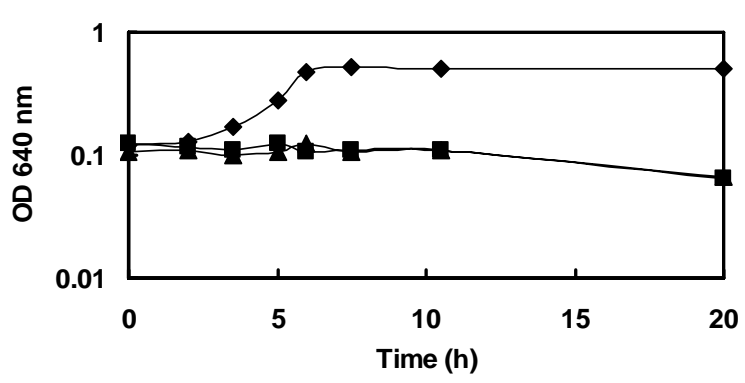

Fig. 4. Growth curves obtained in YCB and YNB containing 5mM metanilic acid ( $\mathbf{\Delta})$ or $5 \mathrm{mM}$ sulfanilic acid ( $\square)$; controls $(\bullet)$ were done in the presence of $2.5 \mathrm{mM}$ ammonium sulphate (in YCB) or 5mM glucose (in YNB).

\subsection{Effect of cell pre-adaptation on decolourisation rates}

In order to obtain some evidence regarding the constitutive or inducible nature of the azo reductase activity, the decolourisation rates by cells pre-adapted to the dyes were compared with those observed in cultures inoculated with cells grown in a medium without dye but, otherwise, of identical composition. The results displayed in Fig. 5 clearly show that azo bond reduction is mediated by some constitutive reductase since, in every case, the decolorization progress is virtually independent on cell preadaptation. The observed differences in the half decolourisation times, which range from 1.5-4.5 h, are therefore related to dye structure. 
Dye I

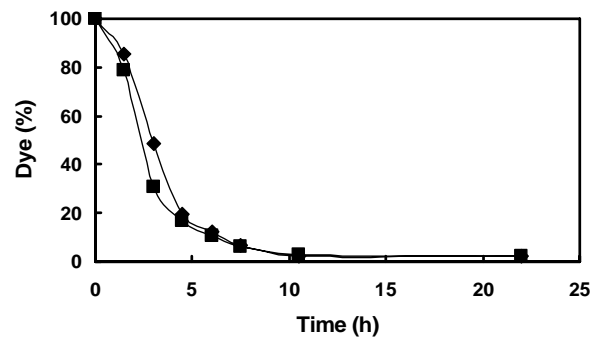

Dye III

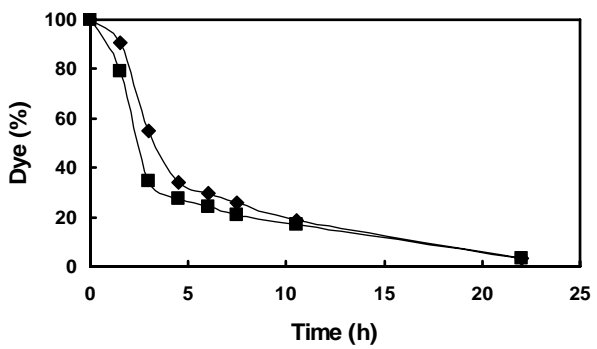

Dye II

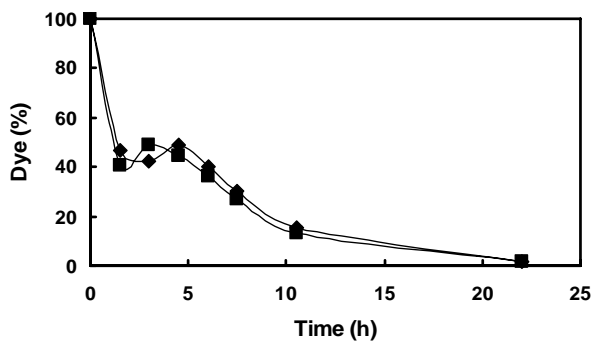

Dye IV

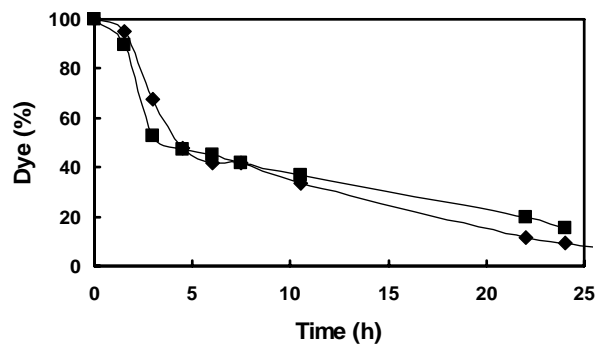

Fig. 5. Time-course of the decolourisation of Dyes I-IV by pre-adapted ( $\square$ ) and nonadapted $(\diamond)$ cells of $C$. zeylanoides (for details see text).

\subsection{Structural effects on decolourisation rates}

Azo dye reduction in the presence of intact cells, by a redox shuttle mechanism, is expected to be an apparent zero-order reaction, while cells are actively growing, and reduced cofactors are abundant. This seems to be the case with the yeast strain used in this work. In fact, following a short latency phase, observed with Dyes I, III and IV, dye concentration decreases linearly with time, at least in the first half of the decolorization process (Fig. 5). The apparent decolourisation rates for dyes I-IV, estimated in the linear portion of the decolourisation curves, and referred to initial dry cell weight, are shown in table 2 . 
Table 2

Apparent decolorization rates

\begin{tabular}{ll}
\hline Dye & Rate $\left(\mu \mathrm{mol} \cdot \mathrm{g}^{-1} \cdot \mathrm{h}^{-1}\right)$ \\
\hline I & 23 \\
II & 52 \\
III & 18 \\
IV & 14 \\
\hline
\end{tabular}

Interestingly Dyes I, III and IV have very similar apparent zero-order decolorization rates, but significantly lower than the observed with Dye II. These results are, so far, intriguing, even taking into account some of the factors which are likely to affect decolourisation rates, as the steric and electronic characteristics of the substrate, the redox potential of the mediator in relation to that of the azo compound, or the specificity of the reductase towards the mediator. Indeed, in Dyes III and IV, which share the same coupling component (2-naphthol), the position of the sulphonic substituent in the diazo component does not significantly affect the decolorization rate. In contrast, in Dyes I and II, where the only structural difference is also the position of the sulphonic substituent, those rates are rather different. One hypothesis which could account for the (so far) unexplained differences in decolourisation rates is the occurrence of distinct reduction mechanisms, depending on the particular dye substrate. Two possible mechanisms for complete azo reduction are a $\mathrm{NAD}(\mathrm{P}) \mathrm{H}$-like reduction, involving two steps of hydride transfer, each preceded (or followed) by protonation or, alternatively, a flavin-like reduction, where four steps of $\mathrm{H}^{+}+\mathrm{e}^{-}$addition would take place. Quite likely the limiting rates in each mechanism would be differently affected by structural features of the substrates. This hypothesis might also account for other unexplained observations as those of Semdé et al. [17] who reported that a NADPH generator system, while being able to reduce the dye amaranth, did not reduce methyl orange or orange II (Dyes II and IV in this study), among other dyes. Also Nam and Renganathan [26], in their study of the non-enzymatic reduction of azo dyes by NADH, were not able to establish a simple correlation between the electronic properties of the substituents in 4-(4'-sulfophenylazo)-phenol derivatives, or their position relatively to the azo bond, and the decolourisation extent, after a fixed time. It thus appears that a 
suitable basis for correlating azo reduction rates and dye structures has not yet been found.

\section{Conclusions}

The present work described improved conditions for the decolourisation of several structurally-related azo dyes by a strain of the yeast $C$. zeylanoides. Apparently, the major factor determining the shorter decolourisation times now described is the composition of the incubation medium. Decolourisation is due to azo bond reduction, as shown by the detection of the corresponding amines in the supernatants of cultures where dye decolorization was taking place, and occurs at an acidic $\mathrm{pH}$. The sulphonated amines produced by reduction of the tested dyes could be used as nitrogen sources, but not as carbon and energy sources by the yeast strain. The azo reductase activity towards each dye does not depend on pre-adaptation of the cells to that dye and is therefore due to an unidentified reductase which is constitutive to the yeast strain under the cultivation conditions reported in this work. The available evidence suggests that dye structure affects decolourisation rates but the basis for establishing a correlation between those two parameters remains, so far, unknown.

\section{Acknowledgements}

This work was partially funded by JNICT/Praxis XXI/2/2.2/QUI/44/94 (Portugal) and by the University of Minho. H.S. was a student from the Graz University of Technology (Austria) doing an individual project at the Chemistry Department, University of Minho, supported by the Erasmus programme.

\section{References}

[1] Zollinger, H, Colour Chemistry: Synthesis, Properties and Applications of Organic Dyes and Pigments, VCH Publishers, Inc., NY, 1987, 92-102.

[2] Pagga, U. and Brown, D. The degradation of dyestuffs II. Behaviour of dyestuffs in aerobic biodegradation tests. Chemosphere 1986, 15, 479-491.

[3] Shaul, G.M., Holdsworth, T.J., Dempsey, C.R. and Dostal, K.A. Fate of water soluble azo dyes in the activated sludge process. Chemosphere 1991, 22, 107-109. 
[4] Chung, K.-T. and Stevens Jr., S.E. Degradation of azo dyes by environmental microorganisms and helminths. Environ. Toxicol. Chemistry, 1993, 12, 2121-2132.

[5] Pierce, J. Colour in textile effluents-the origins of the problem. J. Soc. Dyers Colourists 1994, 110, 131-134.

[6] Dubrow, S.F., Boardman, G.D. and Michelsen, D.L. Chemical pretreatment and aerobic-anaerobic degradation of textile dye wastewater. In: Environmental Chemistry of Dyes And Pigments (A. Reife \& H.S. Freeman, Eds). John Wiley \& Sons, NY, 1996, 75-104

[7] Chung, K.-T., Stevens Jr., S.E. and Cerniglia, C.E. The reduction of azo dyes by the intestinal microflora. Crit. Rev. Microbiol. 1992, 18, 175-190.

[8] Chung, K.-T. The significance of azo-reduction in the mutagenesis and carcinogenesis of azo dyes. Mut. Res. 1982, 114, 269-281.

[9] $\mathrm{Hu}, \mathrm{T} . \mathrm{L}$. Kinetics of azoreductase and assessment of toxicity of metabolic products from azo dyes by Pseudomonas luteola. Water Sci. Technol. 2001, 43, 261-269.

[10] Zimmermann, T., Kulla, H.G. and Leisinger, T. Properties of purified Orange II azoreductase, the enzyme initiating azo dye degradation by Pseudomonas KF46. Eur. J. Biochem. 1982, 129, 197-203.

[11] Zimmermann, T., Gasser, F., Kulla, H.G. and Leisinger, T. Comparison of two bacterial azoreductases acquired during adaptation to growth on azo dyes. Arch Microbiol. 1984, 138, 37-43.

[12] Rafii, F., and Coleman, T. Cloning and expression in Escherichia coli of an azoreductase gene from Clostridium perfringens and comparison with azoreductase genes from other bacteria. J. Basic Microbiol. 1999, 39, 29-35.

[13] Chang, J.S., Kuo, T.S., Chao, Y.P., Ho, J.Y. and Lin, P.J. Azo dye decolorization with a mutant Escherichia coli strain. Biotech. Lett. 2000, 22, 807-812.

[14] Suzuki, Y., Yoda T., Ruhul, A. and Sugiura, W. Molecular cloning and characterization of the gene coding for azoreductase from Bacillus sp. OY1-2 isolated from soil. J Biol Chem. 2001, 276, 9059-9065.

[15] Keck, A., Klein, J., Kudlich, M., Stolz, A., Knackmuss, H.J. and Mattes, R. Reduction of azo dyes by redox mediators originating in the naphthalenesulfonic acid degradation pathway of Sphingomonas sp. strain BN6. Appl Environ. Microbiol. 1997, 63, 3684-3690. 
[16] Kudlich, M., A. Keck, J. Klein and A. Stolz. Localization of the enzyme system involved in anaerobic reduction of azo dyes by Sphingomonas sp. strain BN6 and effect of artificial redox mediators on the rate of azo dye reduction. Appl. Environ. Microbiol. 1997, 63, 3691-3694.

[17] Semdé, R., Pierre, D., Geuskens, G., Devleeschouwer, M. and Moës, A.J. Study of some important factors involved in azo derivative reduction by Clostridium perfringens. Internat. J. Pharm. 1998, 161, 45-54.

[18] Ogawa, T., Yatome, C. and Idaka, E. Biodegradation of $p$-aminoazobenzene by continuous cultivation of Pseudomonas pseudomallei 13NA. J. Soc. Dyers Colourists, 1981, 97, 435-438.

[19] Idaka, E., Ogawa, T. and Horitsu, H. Reductive metabolism of aminoazobenzenes by Pseudomonas cepacia. Bull. Environ. Contam. Toxicol. 1987, 39,100-107

[20] Robinson, T., McMullan, G., Marchant, R. and Nigam, P. Remediation of dyes in textile effluent: a critical review on current treatment technologies with a proposed alternative. Bioresour. Technol. 2001, 77, 247-255

[21] Stolz, A. Basic and applied aspects in the microbial degradation of azo dyes. Appl. Microb. Biotechnol. 2001, 56, 69-80.

[22] Martins, M.A., Cardoso, M.H., Queiroz, M.J., Ramalho, M.T. and Campos, A.M. Biodegradation of azo dyes by the yeast Candida zeylanoides in batch aerated cultures. Chemosphere 1999, 38, 2455-2460.

[23] Miller, G. L. Use of dinitrosalicylic acid reagent for determination of reducing sugar. Anal. Chem. 1959, 31, 426-428.

[24] Lages, F. and Lucas, C. Contribution to the physiological characterization of glycerol active uptake in Sacccharomyces cerevisiae. Biochim. Biopshys. Acta 1997, 1322, 8-18.

[25] Chang, J.-S., Chou, C., Lin, Y.-U., Lin, P.-J., Ho, J.-Y. and Hu, T.L. Kinetic characteristics of bacterial azo-dye decolorization by Pseudomonas luteola. Wat. Res. 2001, 35, 2841-2850.

[26] Nam, S. and Renganathan, V. Non-enzymatic reduction of azo dyes by NADH. Chemosphere, 2000, 40, 351-357. 
[27] Kudlich, M., Hetheridge, M.J., Knackmuss, H.-J. and Stolz, A. Autoxidation reactions of different aromatic $o$-aminohydroxynaphthalenes that are formed the anaerobic reduction of azo dyes. Environ. Sci. Technol. 1999, 33, 896-901.

[28] Gottschalk, G. and Knackmuss, H.-J. Bacteria and the biodegradation of chemicals achieved naturally, by combination, or by construction. Angew. Chem. Int. Ed. Engl. 1993, 32, 1398-1408.

[29] Nörtemann, B., Baumgarten, J., Rast, G. and Knackmuss, H.-J. Bacterial communities degrading amino and hydroxynaphthalene-2-sulfonates. Appl. Environ. Microbiol. 1986, 52, 1195-1202.

[30] Blümmel, S., Contzen, M., Lutz, M., Stolz, A. and Knackmuss, H.-J. Isolation of a bacterial strain with the ability to utilize the sulfonated azo compound 4-carboxy4'-sulfoazobenzene as the sole source of carbon and energy. Appl. Environ. Microbiol. 1998, 64, 2315-2317. 